\title{
Mondes du tourisme : un projet scientifique renouvelé
}

Mathis Stock

\section{(2) OpenEdition}

\section{Journals}

Édition électronique

URL : http://journals.openedition.org/tourisme/2291

DOI : 10.4000/tourisme.2291

ISSN : 2492-7503

Éditeur

Éditions touristiques européennes

\section{Référence électronique}

Mathis Stock, «Mondes du tourisme : un projet scientifique renouvelé », Mondes du Tourisme [En ligne], 15 | 2019, mis en ligne le 01 juin 2019, consulté le 25 septembre 2020. URL : http:// journals.openedition.org/tourisme/2291 ; DOI : https://doi.org/10.4000/tourisme.2291

Ce document a été généré automatiquement le 25 septembre 2020

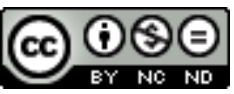

Mondes du tourisme est mis à disposition selon les termes de la licence Creative Commons Attribution - Pas d'Utilisation Commerciale - Pas de Modification 4.0 International. 


\title{
Mondes du tourisme : un projet scientifique renouvelé
}

\author{
Mathis Stock
}

1 Le tourisme est marqué par de nouveaux enjeux qui affectent le positionnement scientifique de Mondes du tourisme, revue de recherche qui affirme que «le tourisme peut être construit comme objet de connaissance scientifique isolable dans le flux des événements et processus des sociétés humaines ». Ces enjeux concernent tant le développement des sciences humaines et sociales que les changements sociétaux.

2 Tout d'abord, le tournant mobilitaire qui marque actuellement les sciences humaines et sociales (SHS) a des effets sur les études du tourisme en raison de l'interdépendance entre les mobilités touristiques et toutes les autres formes de mobilité. La question des modes de vie mobiles, de la multilocalité ou polytopicité de la vie humaine permet d'appréhender le tourisme comme étant articulé, et non séparé, d'autres modes d'être mobiles ou immobiles. Ensuite, le spatial turn des SHS, centré sur les spatialités relationnelles et l'attention donnée au place making, à l'invention et à la construction sociale des lieux touristiques a également infusé dans les études du tourisme. Enfin, l'avènement des digital humanities et la montée en puissance des STS (sciences, technologies et société) font comprendre la nécessité d'engager un travail plus approfondi sur l'infrastructure numérique du tourisme, les nouvelles pratiques touristiques associées aux usages des instruments numériques, voire l'assouplissement de la distinction entre quotidien et hors-quotidien par les réseaux sociaux numériques.

3 Les nouveaux enjeux sociétaux concernent le lien à la durabilité, avec la crise politique autour du changement climatique, mais aussi les controverses touristiques dans des villes, liées entre autres aux technologiques numériques; mais également les crises liées au néolibéralisme, dont les effets sur les inégalités, la fuite des capitaux et les crises financières sont à investiguer. Enfin, la modification de la composition des touristes à l'échelle mondiale - toujours plus de touristes issus des "Suds » et des « Nords »-s'accompagne d'une crise migratoire - qui fait toucher du doigt la sélectivité et les différentes valeurs assignées aux différentes formes de mobilité. Après une longue phase de célébration du tourisme en tant qu'élément de la mondialisation, on 
semble amorcer un mouvement de dé-mondialisation, de la proximité, des circuits courts - des controverses qui touchent aussi le tourisme.

4 La position de Mondes du tourisme dans le champ des revues scientifiques est aussi dépendante du changement de rapport de force dans l'économie de la connaissance actuelle. D'une part, l'injonction à la publication rapide, à l'opposé de la slow science, à l'excellence ( $c f$. la charte de la dés-excellence) et à la compétition, opposée à la coopération, a créé un « marché saturé » où tout le monde écrit et plus personne ne lit. D'où l'ambition de Mondes du tourisme de publier des articles de qualité et de ne pas céder à la fast science. D'autre part, le rayonnement de la recherche francophone passe aussi par l'intervention dans le contexte international, et Mondes du tourisme vise à publier davantage d'articles en anglais, lingua franca des échanges scientifiques contemporains.

5 Dans ce contexte, la ligne éditoriale renouvelée de Mondes du tourisme engage la vision suivante :

- Recherches théorico-empiriques. Les recherches empiriques sont cadrées par des éléments conceptuels à travers lesquels la recherche poursuit des finalités théoriques. Que ce soit par des méthodes quantitatives et/ou qualitatives, la production et l'analyse de données originales est en lien avec des éléments conceptuels, et non des articles uniquement empiriques. Que cela relève de la grounded theory ou de la theory-laden observation, peu importe! Des pièces purement théoriques qui avancent ou discutent des fondements conceptuels sont également les bienvenues.

- Méthodologies. Nous publions aussi des articles purement méthodologiques, car les réflexions méthodologiques et la production de nouvelles méthodologies restent sous-développées à l'heure actuelle. Que ce soit dans les méthodes quantitatives ou qualitatives, l'innovation conceptuelle (mobile methods, ethnographie du web et des algorithmes, nouvelles quantifications, etc.) paraît plus nécessaire que jamais.

- Inter- et transdisciplinarité. Il s'agit d'une revue pluridisciplinaire autour d'un objet d'études, le tourisme, analogue aux urban studies, digital studies, gender studies, etc., à l'étude duquel concourent potentiellement toutes les disciplines. Aucune approche n'est exclue a priori. Ceci donne une responsabilité aux auteurs, comme au comité de rédaction, de rendre explicite les perspectives adoptées, mais permet aussi des visions inédites par le croisement de plusieurs disciplines, voire l'indiscipline...

- Penser par et avec le tourisme. L'objectif n'est pas seulement de dire quelque chose de sensé ou de critique sur le tourisme, mais aussi de comprendre comment les enjeux touristiques sont présents dans les problèmes sociaux. D'où l'ambition de la revue de diffuser des recherches qui entrent par le tourisme pour aborder des questions plus larges. Le tourisme comme perspective pour comprendre le fonctionnement, les crises, les transformations des sociétés humaines peut ainsi être un moyen, et non un but en soi. Virtuellement toutes les recherches en SHS rencontrent le tourisme, c'est pourquoi nous encourageons aussi à publier les recherches qui ne touchent le tourisme qu'à la marge pour évoquer les enjeux centraux des sociétés humaines.

- Critique. Une critique du tourisme, mais aussi une critique de la critique du tourisme doit être engagée. Dans un contexte de valeurs clivantes associées au tourisme entre le tourisme comme élément d'autonomie voire de liberté, de cosmopolitisme, et, au contraire, le tourisme comme destructeur des milieux géographiques, authenticités, rapports sociaux et étant porté par des «idiots du voyage » (Urbain, 1991), les recherches publiées engagent la 
difficile tâche d'analyser le tourisme et les discours portant sur celui-ci avec détachement scientifique.

- Les marges du tourisme. L'idéal-type des pratiques touristiques continue à exister, mais les multiples mobilités, digitalités et résidentialités font émerger des pratiques nouvelles qui étirent le champ du tourisme. Thématiquement, la revue reste centrée sur le tourisme, mais - prenant acte du mobilities turn et de l'absence de revue francophone dédiée aux mobilités vise aussi la diffusion de recherches sur les multiples formes de mobilité dans lesquelles le tourisme n'apparaîtrait qu'à la marge.

6 C'est dans ce contexte de changement que le comité scientifique de la revue Mondes du tourisme se renouvelle aujourd'hui. C'est pour nous l'occasion de remercier l'équipe sortante pour son engagement depuis le début de l'aventure : les professeurs Cécile Clergeau, Philippe Duhamel, Jean-Pierre Poulain, Laurent Tissot, Philippe Violier et Vincent Vlès ont contribué au succès de la revue, d'abord dans sa forme "papier ", puis, depuis 2015, en tant que revue en ligne. Le professeur Philippe Violier doit être tout particulièrement cité qui, en tant que rédacteur en chef, a porté la revue sur les fonts baptismaux en 2010 et a ainsi assuré un rayonnement des études sur le tourisme en langue française.

7 Une nouvelle équipe est à l'œuvre pour poursuivre la publication et la diffusion de recherches scientifiques sur les dimensions touristiques des sociétés humaines, sous tous leurs aspects.

\section{AUTEUR}

\section{MATHIS STOCK}

Rédacteur en chef de Mondes du tourisme 